\title{
Lidil
}

Revue de linguistique et de didactique des langues

\section{Faraco M., La classe de langue. Théories, méthodes et pratiques}

2006, Aix-en-Provence, Publications de l'université de Provence.

\section{Omar Colombo}

\section{OpenEdition}

\section{Journals}

Édition électronique

URL : http://journals.openedition.org/lidil/38

DOI : $10.4000 /$ lidil.38

ISSN : 1960-6052

\section{Éditeur}

UGA Éditions/Université Grenoble Alpes

Édition imprimée

Date de publication : 1 décembre 2006

ISBN : 2-914176-15-5

ISSN : $1146-6480$

\section{Référence électronique}

Omar Colombo, «Faraco M., La classe de langue. Théories, méthodes et pratiques », Lidil [En ligne], 34 | 2006, mis en ligne le 01 octobre 2007, consulté le 21 septembre 2020. URL : http:// journals.openedition.org/lidil/38 ; DOI : https://doi.org/10.4000/lidil.38

Ce document a été généré automatiquement le 21 septembre 2020

(C) Lidil 


\title{
Faraco M., La classe de langue. Théories, méthodes et pratiques
}

\author{
2006, Aix-en-Provence, Publications de l'université de Provence.
}

Omar Colombo

1 Les auteurs de La classe de langue s'intéressent à la didactique des langues et à la recherche sur l'acquisition d'une langue seconde. Ils défendent l'idée selon laquelle la classe de langue est l'endroit parfait pour le recueil de données nécessaires à tous ceux qui travaillent en apprentissage de langue seconde.

2 Dans le premier chapitre («Enseignement de langue et langue d'enseignement »), JeanMarc Defays et Sarah Deltour (13-27) mettent en évidence les spécificités et les paradoxes de la classe de langue. Si au début de l'histoire de l'enseignement d'une langue la méthodologie adoptée était très similaire à celle de l'enseignement d'autres matières, l'évolution des méthodologies a porté l'enseignement d'une LE d'une part à avoir ses propres spécificités, d'autre part à le faire devenir l'objet d'observation de chercheurs et d'enseignants, chacun prêtant une attention particulière selon ses propres intérêts scientifiques. Après un "bref historique » (15-17) des méthodologies employées par les didacticiens des langues, du béhaviorisme des années quarante à l'approche communicative des années quatre-vingt, les auteurs passent en revue «les contenus » de l'enseignement des langues, c'est-à-dire le discours et la communication (18-20). Dans l'enseignement d'une langue, les professeurs doivent faire preuve de plusieurs compétences : ils doivent connaître le niveau des apprenants, leurs capacités, leurs faiblesses en adaptant ainsi au mieux, leur pédagogie. L'observation de classe peut réellement aider l'évolution des pédagogies d'après les nécessités contextuelles. Quant aux « objectifs » à devoir atteindre (23-24), on est passé de la préférence pour le savoirfaire, à l'analyse des erreurs, jusqu'à l'interculturel et le savoir-être. Face à cette multitude d'intérêts, il devient primordial d'enquêter sur les priorités réelles des apprenants. L'observation est, enfin, essentielle pour pouvoir évaluer les compétences des apprenants. Il s'agit d'une activité à mettre en place par l'enseignant qui doit faire appel à son intuition pour rendre compte des innombrables compétences de l'apprenant (cognitives, relationnelles, métalinguistiques...) : " les recherches et 
observations n'ont toujours pas apporté à ces questions les réponses qui permettraient d'élaborer une évaluation réellement communicative [...] (Defays/Deltour, 25). Gérald Schlemminger et Claude Springer (29-43) comparent la classe de langue à la classe où l'on enseigne les matières non linguistiques: il semblerait que les apprenants d'une langue étrangère peuvent perfectionner leur acquisition grâce, principalement, à la planification didactique. D'après les auteurs, seule une nouvelle approche bi et plurilingue permettra d'améliorer et de faire évoluer les stratégies communicatives.

Dans le deuxième chapitre ("Pistes théoriques »), Pierre Bange (47-70) souligne l'idée de Vygotski d'après laquelle l'apprentissage d'une langue seconde et celui d'une langue maternelle partagent les mêmes processus. Les différences entre les deux apprentissages sont à rechercher dans les conditions internes (liées à l'histoire individuelle du sujet et jouant un rôle primordial pour la L1) et externes (les conditions interactionnelles de l'apprentissage, et en particulier dans une situation de classe, essentielles pour la L2). Quant à la "vérification empirique des relations entre L1 et L2» (52-56) c'est, d'après l'auteur, au niveau du processus métacognitif que la L1 semble bien jouer un rôle de médiation à la L2 : «dans l'approche connexionniste, les processus cognitifs sont compris en termes de réseaux. La mémoire sémantique est organisée comme les mailles d'un filet avec de nombreuses interconnexions [...] le stockage en mémoire est adressable par ses contenus; ce qui signifie qu'un attribut (par exemple la couleur d'un objet) peut être utilisé pour localiser le matériel en mémoire " (Bange, 56-57). La dénomination d'un concept en L1 aiderait l'activation de la recherche d'un objet moins calibré, par exemple sa dénomination en L2. Ainsi, les relations entre L1 et L2 sont comprises en termes de relations entre réseaux. En passant par le modèle des trois types de bilinguisme de Weinreich, le bilinguisme composé, subordonné et coordonné (Weinreich, 1953 cité par Bange, 58-62), Bange tient compte des conditions internes pour arriver à la conclusion que le recours cognitif à la L1 et, inversement, la dépendance cognitive de la L2 à la L1, s'affaiblissent au fur et à mesure que l'apprentissage progresse, et cela grâce à ce que l'auteur définit comme le processus de procéduralisation (Bange, 61). Parmi «les conditions externes» (62-66), et particulièrement en ce qui concerne l'interaction de classe, l'interaction est, en soi, mise au centre de l'activité pédagogique de la part du tuteur (l'enseignant) : l'activité cognitive de l'élève est censée se réduire à la seule dimension d'apprentissage que lui dicte l'enseignant. De ce point de vue Bange incite le didacticien à prendre en compte le côté cognitif de l'apprenant qui n'est nullement indépendant de l'apprentissage en soi. Il invite également à ne pas perdre de vue l'approche communicative. Selon l'auteur, c'est seulement si nous tenons compte des variables internes et externes que nous pourrons bâtir une didactique efficace, communicative et cognitive. Ulrich Dausendschön-Gay (71-91) nous présente les résultats d'une expérience francoallemande à l'école primaire : l'auteur s'intéresse aux « dynamiques de communication triangulaire et autogérée » (Faraco, 7) et à leur importance dans l'acquisition initiale d'une LE. Tsuyoshi Kida (93-110) analyse le geste en tant qu'objet d'observation de la communication non-verbale en classe de langue ; d'après l'auteur, afin que l'on puisse étudier et enseigner le non-verbal, il faut d'abord planifier la méthode d'analyse, et prédéfinir l'objet à enseigner.

4 Dans le troisième chapitre ( Le point de vue des acteurs, motivations, attitudes et représentations »), Sonja Janssens, Alex Housen et Michel Pierrard observent de près à Bruxelles la perception d'apprenants francophones et néerlandophones de deux L1 et/ ou L2, le français et le néerlandais (113-127). Quant aux « attitudes envers le contexte 
d'apprentissage » (120-122), les étudiants francophones semblent apprécier favorablement les cours autant du néerlandais L2 que du français L1, tandis que les néerlandophones paraissent généralement plus neutres. Les facteurs qui justifieraient ces résultats sont au nombre de trois : le facteur temps (les néerlandophones L1 comme les francophones L2 passent d'une perception positive à une perception plus neutre entre la première et la troisième année : l'enthousiasme s'affaiblit avec le temps), le facteur âge (le cours et le professeur de néerlandais L1 sont évalués plus favorablement par les 15-17 ans que par les 13-15 ans), le facteur L1 (le cours et le professeur de néerlandais L1 présentent une perception favorable généralisée, tandis que les néerlandophones sont moins favorables que les francophones envers l'enseignement du français). Et il en est de même pour la "motivation envers l'appropriation " (122-125) : l'âge et la L1 semblent confirmer leur importance dans les motivations et les perceptions des acteurs, tandis que le facteur temps perdrait de son influence (les apprenants néerlandophones sont autant motivés que les francophones dans l'apprentissage des L2 tout au long du processus d'acquisition). Nous retrouvons des différences de perception vis-à-vis de l'italien LM et de l'anglais LE dans l'analyse qualitative et quantitative de logonymes effectuée auprès d'enseignants et d'élèves par Antonietta Marra et Gabriele Pallotti (129-151) : les perceptions sont significativement divergentes. Jean-Marc Dewaele et Gaëlle Planchenault (153-171), quant à eux, analysent la perception et l'utilisation des pronoms d'adresse français tu et vous: plus l'étudiant progresse dans son apprentissage du français et plus il ressent des difficultés dans le choix linguistique des deux pronoms. En conclusion, les deux auteurs estiment que plus la proximité entre la L1 et la L2 est majeure, plus les apprenants seront à l'aise dans l'apprentissage de la LE.

5 Les auteurs $\mathrm{du}$ quatrième chapitre traitent plusieurs questions concernant l'enseignement d'une LE, comme l'indique bien son titre: "Perspectives ciblées. Sociolinguistique, Discours et syntaxe, Littérature, Phonétique et Non-verbal ». Sophie Babault et Rada Tirvassen (175-198), en critiquant la conceptualisation d'une grammaire descriptive comme pivot de la didactique d'une langue L1 et/ou L2, défendent la priorité à donner à la prise en compte des facteurs sociolinguistiques de la part de la didactique en classe de langue, pendant les interactions verbales et l'évaluation des compétences des apprenants. Quant aux «outils sociolinguistiques pour l'observation de la classe de langue » (177-186), les auteurs revisitent d'abord la notion de répertoire langagier de Gumperz et, à partir de là, les notions de plurilinguisme, de contact de langues et d'alternance codique, d'identité, de pédagogie communicationnelle et d'actes de langage qui en découlent: "l'analyse des phénomènes d'alternance de langue en classe montre [...] la façon dont les apprenants jouent sur l'ensemble de leur répertoire afin de mettre en œuvre des stratégies d'appropriation de la langue cible [...] " (Babault/Tirvassen, 179-180). La sociolinguistique se place également au centre de la didactique des langues lorsqu'elle éclaire deux groupes de notions essentielles. Premièrement, la variation linguistique qui s'affirme face aux principes de langue-modèle et d'uniformité de la langue selon Vaugelas, véhiculés, même à une époque récente, par les grammaires traditionnelles et les dictionnaires: "si l'école exclut les variétés non prestigieuses, elle sanctionne lourdement tout écart par rapport à la variété normée même quand il s'agit d'apprenants de L2 en voie de construire leur système grammatical » (Babault/ Tirvassen, 181). Le deuxième groupe de notions éclairées par la sociolinguistique concerne la diglossie, la représentation et les attitudes personnelles face aux langues, 
phénomènes incontournables des interactions en classe de langue. Après avoir traité brièvement de «la compétence sociolinguistique des apprenants» (186-194), les auteurs terminent leur article en encourageant les écoles à reconnaître d'une part que n'importe quelle langue est soumise à de nombreux facteurs de variation et d'autre part à accepter et intégrer les enseignements de la sociolinguistique dans les pratiques scolaires. Mireille Prodeau (199-214) affirme que pour maîtriser une LE les apprenants doivent d'abord avoir recours à la métalangue et, donc, à l'explicitation des règles de grammaire. L'auteur utilise des recherches en acquisition L2 sur le discours pour créer "des séquences d'apprentissage et provoquer un développement microgénétique des outils syntaxiques en relation avec un discours instructionnel » (Faraco, 8) : dans cette expérience, les apprenants sont amenés à découvrir les éléments grammaticaux et syntaxiques fondamentaux dans l'accomplissement d'une tâche verbale. L'objectif de cette étude est de contenir le plus possible l'émergence de l'accent étranger de l'apprenant. Quant à Brigitte Bonnefoy (215-230), elle défend la valeur de l'usage d'un texte littéraire en didactique des langues. Pour atteindre son objectif, l'auteur rappelle les dix raisons qui soutiendraient la didactisation de la littérature dans un bref compte rendu des recherches scientifiques en la matière. Parmi elles, nous mentionnons à titre d'illustration la littérature comme laboratoire de langue (Brigitte Bonnefoy, 215), dans lequel l'apprenant se trouvera face à une épreuve lexicale difficile, qui d'un côté l'empêchera dans un tout premier temps d'exprimer ses émotions et ses idées, et de l'autre le poussera à abandonner sa langue maternelle : l'apprenant sera ainsi amené à se construire une nouvelle identité linguistique et culturelle. Mais la littérature permet également de présenter la variété des normes linguistiques; de renvoyer l'apprenant au référentiel et donc l'aider à développer son champ communicationnel en améliorant ses compétences interprétatives et sémantiques; de manifester la dimension sensible et esthétique de la langue; de motiver l'imaginaire par le jeu, la fiction et la simulation; de cibler l'interculturel et, de ce fait, la réflexion sur soi et les autres; de construire une nouvelle personnalité identitaire; de faire connaitre le patrimoine culturel et la civilisation de la société. L'auteur prône tout particulièrement l'utilisation de la nouvelle en classe de langue autant pour ses spécificités que pour son adaptation à la lecture en langue étrangère. Bonnefoy termine son article en proposant la didactisation de quelques textes. Danielle Duez et Tomáf Dub 'da (231-248) s'intéressent à l'acquisition des phénomènes d'hypoarticulation du français par des apprenants tchèques. Parmi les faits analysés, les auteurs donnent une priorité particulière, entre autres, au «e » muet, à la nasalisation de voyelles et d'occlusives et à la liaison. Les auteurs analysent d'abord la variabilité des formes sonores d'après la formalité situationnelle: le lieu et le moment de l'interaction sont les deux variables fondamentales capables d'orienter le niveau d'éloignement et de proximité de la communication. L'adaptation à la situation communicationnelle implique d'apprendre à gérer l'hyper et l'hypoarticulation: " chaque situation de communication influence de manière significative la structuration des mouvements et la force articulatoire, qui en retour se reflètent par une information acoustico-phonétique spécifique » (Duez/ Dub'da, 233). Ensuite, il s'agit d'illustrer quelques faits phonétiques et d'hypoarticulation $d u$ français et du tchèque, pour aborder en conclusion une expérience sur la perception et l'acoustique du français par des étudiants tchécophones qui aspirent à devenir des traducteurs : les phénomènes d'assimilation et de réduction ne répondent à aucune régularité observée; elles sont même caractérisées par une vaste variabilité interpersonnelle. Du point de vue de la didactique en classe de langue, 
les auteurs reconnaissent que la reproduction de ces faits phonétiques de la part d'apprenants étrangers, avancés ou pas, ne sera jamais identique à celle de francophones natifs; il est toutefois fondamental de mettre ces phénomènes au centre de l'enseignement pour éviter l'ignorance et, pire, le rejet émotionnel vis-à-vis des difficultés phonétiques qui peuvent en découler. Pierre Durant (249-269) propose quelques pistes pour l'élaboration de cours de phonétique pour des apprenants de niveau avancé à partir de sa propre expérience. L'objectif de Pierre Durant est de les faire travailler sur la compréhension orale du français, en dépit de la tendance répandue en didactique du FLE d'enseigner la phonétique conformément aux méthodes orthoépiques et puristes traditionnelles. Le cours cible la discrimination auditive d'après le contexte communicationnel, dont l'objectif pédagogique est d'améliorer l'anticipation et la décodification du message dans la chaine parlée et la rétroaction à partir de la perception auditive. Toujours dans le domaine de la phonétique, la contribution de Chantal Paboudjian (271-284) illustre un compte rendu des recherches sur la prosodie et tout particulièrement sur l'intonation: «les courants d'analyse des fonctions grammaticale et attitudinale de la prosodie sont ainsi présentés et l'importance des travaux sur la fonction socioculturelle de la prosodie, qui ont révélé l'existence des normes prosodiques propres aux groupes, est soulignée » (Faraco, 9). Si le locuteur étranger espère réaliser une communication en langue cible appropriée, il doit tenir compte des normes existantes à l'égard de la situation interactionnelle et du contexte socioculturel. L'étude longitudinale de Miki Nakahara et Mary-Annick Morel (285-305) vise les stratégies intonatives, gestuelles et morphosyntaxiques mises en actes par des apprenantes japonaises de français: les étudiantes en question ont montré avoir assimilé certaines régularités prosodiques et gestuelles du regard, qui régissent l'interaction en français, avant même l'incorporation des régularités lexicales, morphosyntaxiques et des marques d'hésitation du style « euh ». La mimique de pointage avec les mains, quant à elle, semblerait suivre la tendance à s'atténuer au fur et à mesure que l'apprenante progresse dans son acquisition. Pour finir, Alexis Tabensky (307-329) décrit la gestuelle observée pendant des interactions de classe entre apprenants de FLE.

Le centre d'intérêt des discussions est constitué par l'effort des apprenants à l'aide du langage verbal et gestuel. Ainsi, le geste recouvre le rôle de médiateur discursif et contextuel, ce qui mène l'auteur à mettre la gestualité corporelle au centre de l'attention de la didactique du FLE.

\section{AUTEUR}

OMAR COLOMBO

Université Stendhal-Grenoble 3, Lidilem 